\title{
Sexually Dimorphic Expression and Special Regulatory Sequence of Dnali1 in the Olive Flounder Paralichthys Olivaceus
}

\section{Ling Wang}

Institute of Oceanology Chinese Academy of Sciences

\section{Xungang Tan}

Institute of Oceanology Chinese Academy of Sciences

Congcong Zou

Institute of Oceanology Chinese Academy of Sciences

\section{Lijuan Wang}

Institute of Oceanology Chinese Academy of Sciences

\section{Zhihao Wu}

Institute of Oceanology Chinese Academy of Sciences

\section{Yuxia Zou}

Institute of Oceanology Chinese Academy of Sciences

\section{Zongcheng Song}

Shenghang Aquatic Science and Technology Co. Ltd

Feng You ( $\square$ youfeng@qdio.ac.cn )

Institute of Oceanology Chinese Academy of Sciences

\section{Original Article}

Keywords: dnali1, repeated fragment, sexually dimorphic expression, gonad, 5'UTR, 3'UTR

Posted Date: February 4th, 2021

DOl: https://doi.org/10.21203/rs.3.rs-179353/v1

License: (c) (i) This work is licensed under a Creative Commons Attribution 4.0 International License. Read Full License

Version of Record: A version of this preprint was published at Molecular Biology Reports on April 20th, 2021. See the published version at https://doi.org/10.1007/s11033-021-06342-9. 


\section{Abstract}

Dynein axonemal light intermediate chain 1 (dnali1) is an important part of axonemal dyneins and plays an important role in the growth and development of animals. However, there is little information about dnali1 in fish. Herein, we cloned dnali1 gene from the genome of olive flounder (Paralichthys olivaceus), a commercially important maricultured fish in China, Japan, and Korea, and analyzed its expression patterns in different gender fish. The DNA sequence of the entire gene contained a $771 \mathrm{bp}$ open reading frame (ORF), two different sizes of $5^{\prime}$ untranslated region (5'UTR), and a $1499 \mathrm{bp} 3^{\prime}$ untranslated region (3'UTR). Two duplicated $922 \mathrm{nt}$ fragments were found in dnali1 mRNA. The first fragment contained the downstream coding region and the front portion of 3'UTR, and the second fragment was entirely located in 3'UTR. Multiple alignments indicated that the flounder Dnali1 protein contained the putative conserved domain. Its expression showed sexually dimorphic with predominant expression in the flounder testis, and lower expression in other tissues. The gene with the larger 5'UTR was specifically expressed in the testis. The highest expression level in the testis was detected at stages $\nabla$ and $\nabla$. Transient expression analysis showed that the 922 bp repeated sequence 3'UTR of dnali1 down-regulated the expression of GFP at the early stage in zebrafish. The results implied that dnali1 might play an important role in flounder testis, especially in the period of spermatogenesis, and the 5'UTR and the repetitive sequences in 3'UTR might contain some special regulatory elements for the cilia.

\section{Introduction}

Dyneins, a family of major cytoskeletal motors [1], have wide variety of cellular functions [2] and can be divided into two types, axonemal and cytoplasmic dyneins [3]. They consist of heavy, intermediate, light intermediate, and light chains with different molecular weight and function [4]. The roles of different axonemal dyneins and their assembly processes remain elusive in vertebrates including fish [5]. Dyneins are mainly components of cilium, which have been found in most, if not all, vertebrate organs. Prominent cilia form into sensory structures, the eye, ear, and nose. Cilia are also involved in developmental processes, including left-right asymmetry formation, limb morphogenesis, and the patterning of neurons in the neural tube [6]. Dynein light and intermediate chains are required for the motility of dynein heavy chain and the assembly of axonemal dynein into cilia and flagella [3, 7]. Dynein axonemal light and intermediate chain 1 gene (dnali1) encodes one light-intermediate chain and contains a coiled-coil domain in C-terminal region. Dnali1 has been shown to form complexes with the dynein heavy chains, actin, and caltractin/centrin molecules [8]. In Human (Homo sapiens), DNAL/1 could be a candidate gene for patients suffering from the immotile cilia syndrome [9]. Splice-site mutations of green alga (Chlamydomonas reinhardtii) dnali1 impaired the flagellar motility [8]. However, there is little information about dnali1 in fish. Only a study in zebrafish (Danio rerio) showed that axonemal protein components dnah5 and dnali1 were absent in its primary ciliary dyskinesia with mynd10 mutation [10].

Repetitive sequences are prevalent in genome and have been confirmed to play an important role in biological evolution by regulating gene function [11]. There are about $10 \%$ of all genes containing tandemly duplicated exons in human genome [12]. For example, a duplicated exon of human glycine 
receptor $a-2$ has been noted as a candidate for alternative splicing [12]. Similarly, repetitive sequences in 3' untranslated region (3'UTR) are also important because 3'UTR of an mRNA is essential for many biological activities such as mRNA stability, protein translation, sub-cellular localization, protein binding, and translation efficiency [13]. However, there is almost no relevant report for repetitive sequences of 3'UTR in vertebrates although the studies on function of 3'UTR have been performed. In murine spermatids and mammalian germ cells, transcription and translation of Prm1 were controlled by a conserved element in 3'UTR $[14,15]$. A miR430 recognition binding sequence was found in 3'UTR of zebrafish nanos 3 which could accelerate target mRNA decay after binding $[16,17]$, and the combined effect of codon usage and 3'UTR length determines the stability of maternal mRNAs in the embryos [18].

Olive flounder (Paralichthys olivaceus) is one of the most commercially important cultured marine fish species in Korea, China, and Japan [19]. There are growth differences between female and male individuals, and then study on the flounder sex control and its molecular regulation is valuable. According to our transcriptome data [20], dnali7 was highly expressed in the flounder testis. However, similar to other fish, the detailed data of dnali1 in the flounder are unclear.

In the present study, we cloned the dnali1 and found the repetitive sequences in its 3'UTR. The molecular characteristic expression patterns in the flounder tissues and gonads, and the regulation function of repetitive sequences in 3'UTR were studied. The results will provide basic data for further investigation of the flounder spermatogenesis process.

\section{Materials And Methods}

Fish and sample collection

The wild-type flounders for tissue (25-35 cm total length, $T L)$ and gonadal development (12-47 cm TL) analyses were purchased from Nanshan market (Qingdao, China), temporarily reared in aerated seawater tank at the institute aquarium and fed with commercial particle food twice a day. Twelve tissues (ovary/testis, brain, heart, muscle, head kidney, kidney, intestine, spleen, liver, stomach, gill, and eye) and gonads at development stages $\nabla$ to $\nabla$ were respectively dissected from three male and three female flounders after anesthetization with tricaine methane sulfonate (MS-222, $50 \mathrm{mg} / \mathrm{L}$, Sigma, USA). Parts of the gonads were fixed in Davidsion' fixative for histological section stained with hematoxylin/eosin (HE), which would be used to identify the genders and gonadal development stages [21]. The rest parts of the gonads and other tissues for RNA isolation were frozen as soon as possible and stored in $-80{ }^{\circ} \mathrm{C}$.

Zebrafish (TU strain) were reared in a recirculation culture system at the institute aquarium (temperature $28.5 \pm 1^{\circ} \mathrm{C}$, photoperiod $14 \mathrm{~h}$ light : $10 \mathrm{~h}$ dark). Fish were fed with a commercial particle food twice and brine shrimp once every day. Fertilized eggs were obtained by mixing one male with two female fish in the morning. After washed with the cycling water several times, the eggs were ready for microinjection.

Total RNA extraction and cDNA synthesis 
Total RNA was isolated from the flounder tissue and gonadal samples by using Trizol reagent (TOYOBO, Japan) following the manufacture's protocol. The quantity and purity of the RNA were assessed with electrophoresis in $1 \%$ agarose gel and Nanodrop2000 (Thermo scientific, USA). After DNase I (Thermo scientific, USA) treatment, $1 \mu \mathrm{g}$ total RNA was used for CDNA synthesis with M-MLV reverse transcriptase kit (Promega, USA). The obtained cDNA was preserved at $-20^{\circ} \mathrm{C}$.

Isolation of dnali1 cDNA

Based on the flounder testis and ovary transcriptomic data [20] and the flounder genomic data (Genebank accession no. XM_020093361.1), the flounder dnali1 sequence was cloned and verified. The ORF was cloned through semi-quantitative reverse transcription polymerase chain reaction (RT-PCR) using the flounder testis or ovary cDNA as template and specific primers (dnali1-oF/oR, Table 1). 
Table 1

Primers used in this study

\begin{tabular}{|c|c|c|}
\hline Gene & Sequences $\left(5^{\prime}-3^{\prime}\right)$ & Function \\
\hline dnali1-oF & ATGAGCCCACCAACAGATTCTCTCC & Gene clone \\
\hline dnali1-oR & TCAGCTTTTCTTCGGTGTGGCG & Gene clone \\
\hline 3' gsp1 & GCCCTCAGCAGCCCATTGACGCCTGCC & 3'Race \\
\hline 3'gsp2 & ССАСCTCCACTGAGGCCACCGGGC & 3'Race \\
\hline 5' gsp1 & GCCCGGTGGCCTCAGTGGAGGTGG & 5'Race \\
\hline 5' gsp2 & GGGCAGGCGTCAATGGGCTGCTGAGGC & 5'Race \\
\hline genomic part $A-F$ & TGCCTTTAATCCATATCTTTAACCC & Genomic clone \\
\hline genomic part $A-R$ & TGGGAAAAAGAAAAATCGGAG & Genomic clone \\
\hline genomic part B-F & CTCTACGACCAGTGCTTT & Genomic clone \\
\hline genomic part B-R & CCAATTGGATATTTTAATGA & Genomic clone \\
\hline dnali1-RT-R & GGAGTGCTGGACACTTGC & RT-PCR \\
\hline dnali1RT-F & TCAGCTTTTCTTCGGTGTGGCG & RT-PCR \\
\hline dnali1-5'utr-F & ACATGGGGATGGTATCATTACTCC & RT-PCR \\
\hline$\beta$-actin-RT-F & GGAATCCACGAGACCACCTACA & qPCR/RT-PCR \\
\hline$\beta$-actin-RT-R & TTGCTGATCCACATCTGCTG & qPCR/RT-PCR \\
\hline dnali1-qF & TTCAАТСАСТТСССАААСТ & qPCR \\
\hline dnali1-qR & TCCCTGCGAATGGGACA & qPCR \\
\hline \multirow[t]{2}{*}{ 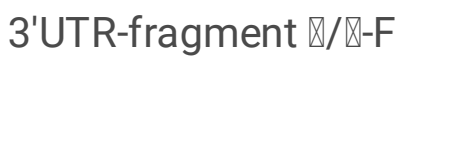 } & GGATGAACTATACAAATAGGGATC & \multirow[t]{2}{*}{ Vector construction } \\
\hline & CAAACTTAAAAACCCTGAGTTACC & \\
\hline \multirow[t]{2}{*}{ 3'UTR-fragment $\otimes-F$} & GGATGAACTATACAAAGGATCCGATGAGCT & \multirow[t]{2}{*}{ Vector construction } \\
\hline & CATCCGACAGGTGACC & \\
\hline \multirow[t]{2}{*}{ 3'UTR-fragment $\otimes / \otimes / \bigotimes-R$} & GGGAGCTCGCCCGGGGATCCCCAATTGA & \multirow[t]{2}{*}{ Vector construction } \\
\hline & TATTTTAAT & \\
\hline
\end{tabular}

The PCR was carried out in a mixture containing $2 \mu \mathrm{L}$ of $\mathrm{cDNA}(50 \mathrm{ng} / \mu \mathrm{L})$ from the flounder testis or ovary, $12.5 \mu \mathrm{L}$ of $2 \times$ GoldStar MasterMix (CWBIO, China), $1 \mu \mathrm{L}$ of forward primer $(10 \mathrm{mM}), 1 \mu \mathrm{L}$ of reverse primer $(10 \mathrm{mM})$, and $8.5 \mu \mathrm{L}$ of RNase-free water. PCR was performed as follows: $95^{\circ} \mathrm{C}$ for $10 \mathrm{~min}, 35$ cycles of $95{ }^{\circ} \mathrm{C}$ for $30 \mathrm{~s}, 55^{\circ} \mathrm{C}$ for $30 \mathrm{~s}$, and $72{ }^{\circ} \mathrm{C}$ for $1 \mathrm{~min}$, and followed by a final extension at $72{ }^{\circ} \mathrm{C}$ for $10 \mathrm{~min}$. 
To obtain 3'UTR and 5'UTR sequences of the flounder dnali1, rapid amplification of cDNA ends (RACE) was performed with specific primers ( $3^{\prime} g s p 1$ and $3^{\prime} g s p 2$, and $5^{\prime}$ gsp1 and 5 'gsp2, Table 1) using SMARTer $^{\text {TM }}$ RACE cDNA Amplification Kit (Clontech, USA) according to the manufacture's protocol. All the PCR products were purified using E.Z.N.A.® Gel Extraction Kit (Omega Bio-Tek, USA). The target fragments were cloned into PEASY-T3 vector (Transgen, China) and sequenced.

Isolation of genomic sequence of the flounder dnali1

The dnali1 genomic sequence was cloned according to genomic sequences provided by Profs. Songlin Chen and Changwei Shao from Yellow Sea Fisheries Research Institute, Chinese Academy of Fishery Sciences. In order to confirm the repeated sequences in dnali1 genomic sequences, specific primers (genomic part A $-F / R$, genomic part $B-F / R$, Table 1) were designed to clone the genomic sequences from three flounder fish (25-35 cm TL). The PCR was carried out in a mixture containing $2 \mu \mathrm{L}$ of genomic DNA $(50 \mathrm{ng} / \mu \mathrm{L})$ from the flounder fins, $12.5 \mu \mathrm{L}$ of $2 \times$ KOD One ${ }^{\text {TM }}$ PCR Master Mix (TOYOBO, Japan), 0.75 $\mu \mathrm{L}$ of forward and reverse primers $(10 \mathrm{mM})$, and $9 \mu \mathrm{L}$ of Dnase/RNase-free water. PCR was performed as follows: $98{ }^{\circ} \mathrm{C}$ for $10 \mathrm{~min}, 35$ cycles of $98{ }^{\circ} \mathrm{C}$ for $10 \mathrm{~s}, 53^{\circ} \mathrm{C}$ for $5 \mathrm{~s}$, and $68^{\circ} \mathrm{C}$ for $20 \mathrm{~s}$, and followed by a final extension at $68{ }^{\circ} \mathrm{C}$ for 5 min. The PCR product was purified and ligated with TOPO-A clone and sequencing.

Transcription factor binding sites prediction

The putative specific transcription factor binding sites in 5'UTR were predicted by online tools program JASPAR (http://jaspar.binf.ku.dk/cgi-bin/jaspar_db.pl) using the JASPAR CORE databases [22].

Multiple alignments and phylogenetic tree construction

Multiple alignments were performed using DNAMAN. The evolutionary history was inferred by using the neighbor-joining method. Evolutionary analyses were conducted using MEGA7 [9].

\section{RT-PCR}

RT-PCR was performed with specific primers (dnali1-5'utr-F and dnali1-oR, Table 1) to test the differential expression of the alternatively spliced 5'UTR of dnali 1 in the flounder testis and ovary at stage $\nabla$.

The expression patterns of dnali1 in the tissues of the adult flounders were evaluated using RT-PCR with specific primers (dnali1-RT-F/R, Table 1). $\beta$-actin ( $\beta$-actin-RT-F/R, Table 1 ) was selected as a reference gene. PCR reaction mixture was exactly the same as described in the "Dnali1 cDNA and $3^{\prime} U T R$ cloning" and the annealing temperatures were $55^{\circ} \mathrm{C}$ for $d$ nali1 and $62{ }^{\circ} \mathrm{C}$ for $\beta$-actin, respectively.

Quantitative expression analysis 
The expression of dnali 1 in the gonads at stages $\nabla-\otimes$ was analyzed using real-time quantitative polymerase chain reaction (qPCR). The templates were prepared using PrimeScript ${ }^{\mathrm{TM}} \mathrm{RT}$ reagent Kit containing gDNA Eraser (Takara, Japan) with $1 \mu \mathrm{g}$ total RNA. According to the manufacturer's instructions, the qPCR was performed using TB Green ${ }^{\text {TM }}$ Premix Ex Taq ${ }^{\text {TM }}$ II (Tli RNase H Plus) (Takara, Japan) in a $20 \mu \mathrm{Lmixture} \mathrm{containing} 10 \mu \mathrm{L}$ of 2xTB Green Premix Ex Taq II, $0.4 \mu \mathrm{L}$ of ROX Reference Dye II, $0.4 \mu \mathrm{L}$ of each forward and reverse primers (dnalit-RT-F/R, Table 1 ), and $1 \mu \mathrm{L}$ of cDNA (50 ng/ $\mu \mathrm{L}$ ), on an Applied Biosystems QuantStudio6 qPCR machine (Life technologies, USA). The PCR was performed as follows, denaturation at $95^{\circ} \mathrm{C}$ for $30 \mathrm{~s}$, followed by 40 cycles of $95^{\circ} \mathrm{C}$ for $5 \mathrm{~s}, 60^{\circ} \mathrm{C}$ for $30 \mathrm{~s}$, and then measured the melting curves. Reaction without template was used as negative controls. $\beta$-actin gene was used as a reference gene ( $\beta$-actin-RT-F/R】 Table 1$)$ in the same condition [23]. Samples were run in triplicates and relative gene expression levels were calculated with the $2^{\wedge-\Delta \Delta \mathrm{Ct}}$ method [24].

Plasmid constructions and microinjection in zebrafish embryos

According to the sequencing results, two 922 bp repeated sequence were found in dnali1 mRNA. To investigate the regulatory activity of the flounder dnali1 3'UTR, different sizes of 3'UTR were cloned into pSP64-GFP vector [25]. Therein, fragment $\otimes$ represented part sequences behind termination codon of the first repeat segment, fragment $₫$ contained the second repeat segment, and fragment $\nabla$ represented whole 3'UTR (fragments $\otimes$ and $\otimes$ ). Three kinds of fragments were subcloned into the $B a m H /$ site that located downstream of GFP coding region in pSP64-GFP vector. The regulating plasmids were constructed using homologous recombination method with SoSoo Cloning kit (TSINGKE, China) and specific primers

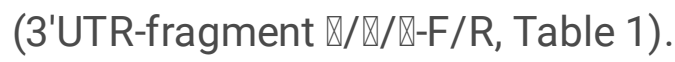

Chimeric RNA composed of GFP coding region and dnali1 3'UTR synthesized by in vitro transcription using a Message Machine SP6 Kit (Ambion, Thermo Fisher Scientific, USA). After purification, the concentration of the capped RNA was diluted to $300 \mathrm{ng} / \mu \mathrm{L}$ in $0.2 \mathrm{M} \mathrm{KCl}$ with phenol red $(0.01 \%)$. This solution (approximately $2 \mathrm{~nL}$ ) was microinjected into the fertilized eggs of zebrafish at one-cell or two-cell stage. The injected eggs were cultured at $28.5 \pm 1{ }^{\circ} \mathrm{C}$. The GFP expression was observed under a fluorescence microscope (Nikon, Japan) at $72 \mathrm{~h}$ post fertilization (hpf).

\section{Data analysis}

One-way analysis of variance with Duncan post hoc test in SPSS 16.0 was used to test significant differences of gene expression among the tissues and gonadal development stages. Student's $t$-test was used to characterize significant differences between the testis and ovary at the same gonadal development stage. The threshold for significance was set as $P<0.05$.

\section{Result}

Characterization of the flounder dnali1 gene 
Genomic sequence for coding region of the flounder dnali1 contained 8 exons and 7 introns (Fig. 1a), and the exon/intron splicing positions were consistent with the GT...AG rule. Coding region was $771 \mathrm{bp}$, which encoded 256 aa. 3'UTR was 1499 bp long (Fig. 1a). There were two alternatively spliced variants in the first exon in 5'UTR (Fig. 1b). It is interesting to find that part of 3'UTR sequence coincided with partial sequence within ORF (Fig. 1). The deducted flounder dnali1 amino acids shared $73 \%, 98 \%$, and $61 \%$ identities with those of Lates calcarifer, Scophthalmus maximus, and Cynoglossus semilaevis. There was a putative conserved axonemal dynein light superfamily domain (pfam10211) in the flounder dnali1 (Fig. 1c). C-terminal region of Dnali1 was more highly conserved, too. Phylogenetic tree showed that the flounder dnali1 was clustered with those from other fish species such as $S$. maximus and L. calcarifer (Fig. 1d).

Confirmation of the large repeated sequence in the genome

About $2.6 \mathrm{~kb}$ fragments of dnali1 genome were respectively cloned from three different flounders (Fig. 2a) and the sequencing results showed that there were two 1229 bp repeat segments. Comparison of the CDNA sequences and the obtained genomic sequences confirmed that there were $1229 \mathrm{bp}$ repeated

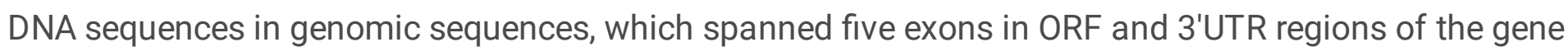
(Fig. 2b).

Sexually dimorphic expression of alternatively spliced 5'UTR of the flounder dnali1

RT-PCR was performed to determine whether alternatively spliced 5'UTR of the flounder dnali1 has male or female specific expression pattern. The results showed that the larger one was only detected in the flounder testis, while the smaller one was found in both the ovary and testis (Fig. 3a). The sequences presented that one SOX5 and one SOX10 binding sites were lost in the smaller one (Fig. 3b).

Tissue distribution of the flounder dnali1

According to the histological analysis, the developmental stages of the testis and ovary of the adult

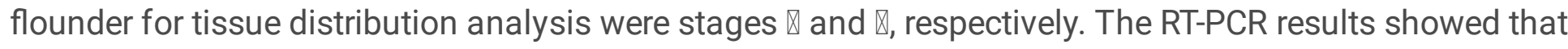
dnali1 was mainly expressed in the testis, while less in the ovary. There was also weak expression in some other tissues or organs such as the male and female brain, heart, eye, and liver (Fig. 4a).

Dnali1 expression at stages $₫-\rrbracket$ of the gonads

Based on the results of the gonadal histological sections (Supplementary Fig. 1), the flounder dnali1 expression at stages $\mathbb{\bigotimes}-\mathbb{Z}$ of gonadal development was tested using qPCR (Fig. 4b). Its expression was

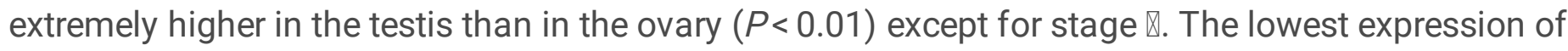

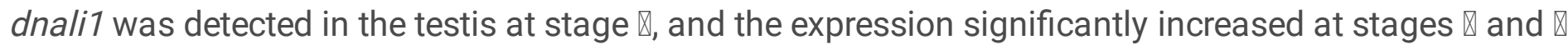
$(P<0.05)$, then it continually significantly increased at stages $₫$ to $\otimes(P<0.05)$. There was no significant difference between stages $\nabla$ and $\nabla$, and $\nabla$ and $\nabla$. The expression levels at stages $\nabla$ and $\nabla$ were

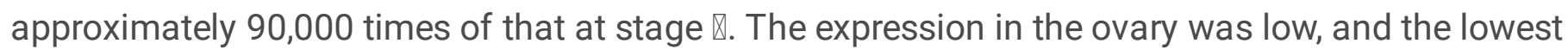

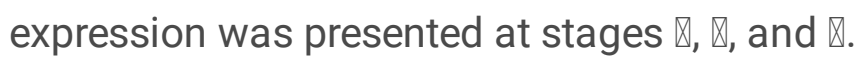


Intriguingly, there were two 922 bp tandem repeated sequences in 3'UTR of dnali1. The first one contained the downstream coding region and front portion of 3'UTR, while the second one was entirely located in 3'UTR. To investigate contribution of the repeated sequence in 3'UTR to the stability and regulation activity of dnali1 mRNA, GFP reporter plasmids containing different sizes of 3'UTR were constructed (Fig. 5a, b) and injected into zebrafish embryos. After hatching, the larvae were analyzed under a fluorescence microscope (Fig. 5c). GFP expression in the fragment $\nabla$ injected group (54/54) was the same as that of the control (59/59). While, there were about $65.91 \%(29 / 44)$ and $52.08 \%(25 / 48)$ of zebrafish injected with the fragments $\nabla$ and $\nabla$ chimeric RNA showing strong GFP expression within heart area, respectively. The expression in the fragment $\nabla$ injected group was stronger than that in the fragment $\nabla$ injected one.

\section{Discussion}

Herein, we isolated and characterized dnali1 gene from the flounder. Its expression in adult tissues and

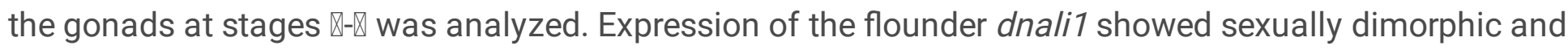
its 3'UTR presented specific RNA retention function.

The flounder dnali1 cloned in this study showed a putative dynein light intermediate chain gene with two splice isoforms of 5'UTR (5'UTR-S, $189 \mathrm{bp}$ and 5'UTR-L, $419 \mathrm{bp}$ ). It is interesting to find that expression of the gene with the larger isoform was male specific. It was estimated that transcripts from $12 \%$ of genes are alternatively spliced within 5'UTRs [26], and these variations in 5'UTR can function as important switches to regulate gene expression $[27,28]$. Transcription factor binding sites and structural motifs in 5'UTR were predicted to analyze the different expression patterns in the male and female flounders. Comparison of the transcriptional factors binding sites, we found that one SOX5 and one SOX10 binding sites were absent in the smaller 5'UTR (5'UTR-S). SOX5 is a transcription factor with homology to the high mobility group box region of the testis-determining factor, SRY. Both of mouse and human SOX5 proteins were only present in tissues containing cells with motile cilia/flagella [29]. SOX10 was expressed in malespecific Sertoli cells only after sex determination in mouse, and required for the maintenance of male fertility in mammal [30]. Expression of dnali 1 was very high at stages $\nabla$ to $\nabla$ in the testis, which suggested that the missed factors of 5'UTR-S, SOX5, and/or SOX10 in the ovary, may be key elements for the regulation of dnali1 expression in the testis. Further study on difference between these two isoforms in regulating activity needs to be performed in the future.

Two sequences of 922 bp in the flounder dnali1 gene were found, which stretched across its ORF region and 3'UTR region. In the genome, repetitive genomic DNA sequences were furtherly confirmed according to the results from three flounder individuals. This phenomenon hasn't been reported in vertebrates so far. To confirm this, dnali1 3'UTR sequences of amphioxus (Branchiostoa floridae) and two flatfish (S. maximus and $C$. semilaevis) were also cloned, sequenced, and blasted, and none of them has repeat sequences (data not shown). Repetitive sequences are prevalent in vertebrate genomes but they are 
usually no more than $100 \mathrm{bp}$ [31]. However, in tick-borne flaviviruses (TBFV), longer repeat sequences (about 200 bp) were found both in 3'UTR and ORF of the genome, which was supposed that 3'UTR might have overlapped function with ORF during the evolution of these viruses [31]. It was implied that multiple duplication of ORF terminal region might be the major event that shaped evolution of the TBFV genome. Tandem gene duplication is usually one of the most prevalent ways of generating genes with new function. Segmental duplication (tandem duplication of a genomic segment) contains both high-copy number repeat and gene sequences with intron-exon structure which occurs frequently to generate redundant genes during evolution [32]. But a minority segmental duplication only produces duplicated exons, rather than entire gene, which is called as tandem exon duplication and is an important source of new exons [33]. The tandem exon duplication is an important mechanism for expanding gene function $[11,34]$. However, wrong tandem exon duplication might also cause negative effect. For example, in human MTM1 mutant genomic DNA, the duplicated MTM1 exon10 produced a 186 base-pair insertion in the MTM1 transcript, which demonstrated there was a necessary intronic sequence for recognition by the spliceosome [35]. The transcript containing the duplicated exon10 retained the reading frame of the wild type transcript and therefore a mutant polypeptide was generated. The repeat segment in the flounder dnali1 is interesting, which duplicated partial genome including one intact exon (E4), one intact intron (14), and a partial exon (partial E5). The first repeated exon (E4) fused with the second repeated exon (partial E5) to form the exon (E5) and the partial exon (partial E5) became new exon (E6). Besides, the first repeat segment included coding region and non-coding region which hasn't been reported in vertebrate before. So, this might be a new kind of evolutionary process. Further studies in more species should be performed to learn evolutionary role of the repeat sequences.

Dynein light intermediate chain protein is a component of the inner dynein arms (IDA), which is important for the assembling of axonemal dyneins [36]. Defects in the axoneme mostly in the structure of outer dynein arms (ODA) and IDA cause primary ciliary dyskinesia [37]. Axonemal abnormalities were observed in $C$. reinharditii flagella if they lacked the dnali1 product [36]. In human, DNALI1 might promote the stable assembly of the dnali7-containing dynein arms or their binding to the axoneme [38]. So, dnali1 could be candidate gene for ciliary motility in mammals. In zebrafish, it has been proved that endothelial primary cilia play a pivotal role for its correct growth and development [39]. In medaka (Oryzias latipes), cilia integrity and axonemal localization of dynein arms were shown to be important for sperm dysmotility, scoliosis, and progressive polycystic kidney [40]. In the present study, dnali1 was mainly expressed in the testis, while in the ovary, its expression kept a low level. The expression levels in the

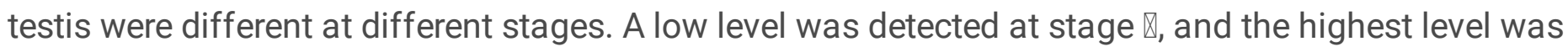

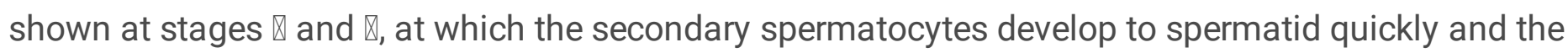
sperm flagella are formed [21]. This high expression results also supported role of dnali1 at these flounder spermatogenesis stages. The sperm axonemal dynein motors consist of ODA and IDA [41]. Splice-site mutations in the gene encoding Dnali1 are correlated in the IDA-4 mutant with a loss of a set of IDA classes, indicating an important role of Dnali1 for the assembly of IDA isoforms [8]. In medaka, uncorrected axonemal localization of dynein arms caused sperm dysmotility [40]. These findings suggest that the dnali1 gene plays an important role in fish spermatogenesis. 
The heart is the first formed organ during embryogenesis [42]. In zebrafish, endocardial primary cilia of the heart, important mediators of fluid flow, play an important role in early embryonic development [43]. Although dnali1 was less expressed in the flounder heart than in the testis, it was required for the motility of cilia and flagella $[3,7]$ and might have function in the flounder heart. It has been proved that 3'UTR of dnali1 not only regulates mRNA-based processes, such as mRNA localization, mRNA stability, and translation, but also transmits genetic information encoded in 3'UTRs to proteins through the establishment of its mediated protein-protein interaction [44]. Zebrafish is an effective model fish to study gene function for its easily observation and microinjection, and has been used in the flounder successfully $[45,46]$. Herein, we analyzed 3'UTR regulation activity of the flounder dnali 1 in zebrafish. The results showed that GFP expression in the fragment $\triangle$ injected group (54/54) was the same as that in the control and fragments $\nabla$ and $\nabla$ injected groups, which could regulate GFP expression in heart and neural tube. The results suggested that the repeated code region (E4) might contain specific regulation elements and its deficiency would decrease mRNA stability, so it didn't show GFP expression in the fragment $\otimes$ injected group. It was suggested that the repeated code region (E4) had dual function, which could control specific translation and maintain RNA stability. Recent study found that 5'UTR and ORF elements and 3'UTR, regulated the translation of Cyclin in mice [47]. In the flounder, 3'UTR might transmit genetic information encoded in 3'UTRs to proteins through the establishment of its mediated proteinprotein interaction. Further studies should be performed to provide evidence to clarify the regulatory mechanism of the repeat sequences.

\section{Conclusion}

In this study, we isolated and characterized dnali1 gene in the flounder. Dnali1 was mainly expressed in

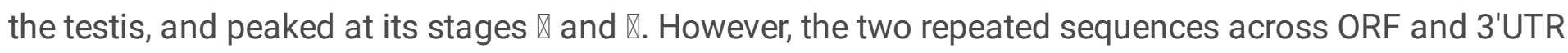
regions of the gene were only found in the flounder and they had different regulation activities. This is the first report about dnali1 gene in marine fish. It will provide evidences for analyzing the flounder spermatogenesis process, as well as cilium development.

\section{Abbreviations}

dnali1, dynein axonemal light intermediate chain 1; ORF, open reading frame; 5'UTR, 5' untranslated region; 3'UTR, 3' untranslated region; TL, total length; MS222, tricaine methane sulfonate; HE, hematoxylin/eosin; RT-PCR, reverse transcription polymerase chain reaction; RACE, rapid amplification of cDNA ends; qPCR, real-time quantitative polymerase chain reaction; IDA, inner dynein arms ; ODA, outer dynein arms.

\section{Declarations}

Acknowledgements 
This work was supported by the projects from the National Sciences of Foundation of China (Nos. 31772834 and 31672636), and the National Key R\&D Program of China (Nos. 2018 YFD0900202 and 2018YFD0901202).

\section{Compliance with ethical standards}

\section{Conflict of interest}

Authors declare no conflict of interests.

\section{Ethical approval}

The study was conducted in accordance with the guidelines and regulations established and approved by the ethics committee of Institute of Oceanology, Chinese Academy of Sciences. All of applicable international, national, and institutional guidelines for the care and use of animals were followed by the authors.

\section{Consent to participate}

All the authors listed have approved the manuscript that is enclosed.

\section{Consent for publication}

The manuscript is approved by all authors for publication.

\section{References}

1. Paschal BM, Vallee RB (1987) Retrograde transport by the microtubule-associated protein MAP 1C. Nature 330:181-183. https://doi.org/10.1038/330181a0

2. Holzbaur ELF, Vallee RB (1994) Dyneins: molecular structure and cellular fnction. Genet Mol Res 10:339-372. http://doi.org/10.1146/annurev.cb.10.110194.002011

3. Cypranowska C, Yildiz A, Ishikawa T (2016) Dyneins. 620-636. https://doi.org/10.1016/B978-0-12394447-4.20101-6

4. King SM (2000) AAA domains and organization of the dynein motor unit. J Cell Sci 113(Pt 14):2521-2526. http://doi.org/10.2307/3583618

5. Yamaguchi $\mathrm{H}$, Oda T, Kikkawa M, Takeda $\mathrm{H}$ (2018) Systematic studies of all PIH proteins in zebrafish reveal their distinct roles in axonemal dynein assembly. Elife 7. https://doi.org/10.7554/eLife.36979

6. Leventea E, Hazime K, Zhao C, Malicki J (2016) Chap. 9 - Analysis of cilia structure and function in zebrafish. In Methods in Cell Biology, H.W. Detrich, M. Westerfield, and L.I. Zon, eds. (Academic Press), pp. 179-227

7. Subramanian A, Kabi A, Gray SF, Pennock D (2016) 28 dynein light chains and ciliary motility in Tetrahymena thermophila. Cytoskeleton 73:197-208. https://doi.org/10.1002/cm.21295 p, . 
8. LeDizet M, Piperno G (1995) The light chain $p 28$ associates with a subset of inner dynein arm heavy chains in Chlamydomonas axonemes. Mol Biol Cell 6:697-711.

https://doi.org/10.1091/mbc.6.6.697

9. Kumar S, Stecher G, Tamura K (2016) MEGA7: molecular evolutionary genetics analysis version 7.0 for bigger datasets. Mol Biol Evol 33:1870-1874. https://doi.org/10.1093/molbev/msw054

10. Zariwala MA, Gee HY, Kurkowiak M, Al-Mutairi DA, Leigh MW, Hurd TW, Hjeij R, Dell SD, Chaki M, Dougherty GW et al (2013) ZMYND10 is mutated in primary ciliary dyskinesia and interacts with LRRC6. Am J Hum Genet 93:336-345. https://doi.org/10.1016/j.ajhg.2013.06.007

11. Babushok DV, Ostertag EM, Kazazian HH (2007) Current topics in genome evolution: molecular mechanisms of new gene formation. Cell Mol Life Sci 64:542-554. https://doi.org/10.1007/s00018006-6453-4

12. Letunic I, Copley RR, Bork P (2002) Common exon duplication in animals and its role in alternative splicing. Hum Mol Genet 11:1561-1567. https://doi.org/10.1093/hmg/11.13.1561

13. Arefeen A, Liu J, Xiao X, Jiang T, Berger B (2018) TAPAS: tool for alternative polyadenylation site analysis. Bioinformatics 34:2521-2529. https://doi.org/10.1093/bioinformatics/bty110

14. Zhong J, Peters AHFM, Kafer K, Braun RE (2001) A highly conserved sequence essential for translational repression of the protamine 1 messenger RNA in murine spermatids 1 . Biol Reprod 64:1784-1789. https://doi.org/10.1095/biolreprod64.6.1784

15. Zhong J, Peters AHFM, Lee K, Braun RE (1999) A double-stranded RNA binding protein required for activation of repressed messages in mammalian germ cells. Nat Genet 22:171-174. https://doi.org/10.1038/9684

16. Giraldez AJ, Mishima Y, Rihel J, Grocock RJ, Van DS, Inoue K, Enright AJ, Schier AF (2006) Zebrafish MiR-430 promotes deadenylation and clearance of maternal mRNAs. Science 312:75-79. http://doi.org/10.1126/science.1122689

17. Sandler H, Stoecklin G (2008) Control mRNA decay by phosphorylation of tristetraprolin. Biochem Soc T 36:491-496. https://doi.org/10.1042/BST0360491

18. Mishima Y, Tomari Y (2016) Codon usage and $3^{\prime}$ UTR length determine maternal mRNA stability in zebrafish. Mol Cell 61:874-885. https://doi.org/10.1016/j.molcel.2016.02.027

19. Fujiwara A, Fujiwara M, Nishida-Umehara C, Abe S, Masaoka T (2007) Characterization of Japanese flounder karyotype by chromosome bandings and fluorescence in situ hybridization with DNA markers. Genetica 131:267-274. http://doi.org/10.1007/s10709-006-9136-z

20. Fan ZF, You F, Wang LJ, Weng SD, Wu ZH, Hu JW, Zou YX, Tan XG, Zhang PJ (2014) Gonadal transcriptome analysis of male and female olive flounder (Paralichthys olivaceus). Biomed Res Int 2014, 291067. http://dx.doi.org/10.1155/2014/291067

21. Sun P, You F, Zhang LJ, Wen AY, Wu ZH, Xu DD, Zhang PJ (2009) Histological evaluation of gonadal differentiation in olive flounder (Paralichthys olivaceus). Marine Sciences 33:53-58. https://doi.org/10.1007/978-1-4020-9623-5_5 
22. Fornes O, Castro-Mondragon JA, Khan A, Lee RVD, Mathelier A (2020) JASPAR 2020: update of the open-access database of transcription factor binding profiles. Nucleic Acids Res 2020;48(D1):D87D92. http://doi.org/10.1093/nar/gkz1001

23. Zheng WJ, Sun $L$ (2011) Evaluation of housekeeping genes as references for quantitative real time RT-PCR analysis of gene expression in Japanese flounder (Paralichthys olivaceus). Fish Shellfish Immun 30:638-645. https://doi.org/10.1016/j.fsi.2010.12.014

24. Livak KJ, Schmittgen TD (2001) Analysis of relative gene expression data using real-time quantitative PCR and the 2 (-Delta Delta C(T)) Method. Methods 25:402-408. https://doi.org/10.1006/meth.2001.1262

25. Li MJ, Tan XG, Jiao S, Wang Q, Wu ZH, You F, Zou YX (2015) A new pattern of primordial germ cell migration in olive flounder (Paralichthys olivaceus) identified using nanos3. Dev Genes Evol 225, 195-206. https://doi.org/10.1007/s00427-015-0503-6

26. Nagasaki H, Arita M, Nishizawa T, Suwa M, Gotoh $O$ (2005) Species-specific variation of alternative splicing and transcriptional initation in six eukaryotes. Gene 364:53-62. https://doi.org/10.1016/j.gene.2005.07.027

27. Barrett LW, Fletcher S, Wilton SD (2012) Regulation of eukaryotic gene expression by the untranslated gene regions and other non-coding elements. Cell Mol Life Sci 69:3613-3634. https://doi.org/10.1007/s00018-012-0990-9

28. Hughes TA (2006) Regulation of gene expression by alternative untranslated regions. Trends Genet 22:119-122. http://doi.org/10.1016/j.tig.2006.01.001

29. Kiselak EA, Shen X, Song J, Gude DR, Wang J, Brody SL, Strauss JF, Zhang Z (2010) Transcriptional regulation of an axonemal central apparatus gene, sperm-associated antigen 6, by a SRY-related high mobility group transcription factor, S-SOX5. J Biol Chem 285:30496-30505. http://doi.org/10.1074/jbc.M110.121590

30. She ZY, Yang WX (2017) Nucleocytoplasmic shuttling of SOX14A and SOX14B transcription factors. Oncotarget 8(29):46955-46968

31. Gritsun TS, Gould EA (2006) The 3' untranslated region of tick-borne flaviviruses originated by the duplication of long repeat sequences within the open reading frame. Virology 354:217-223. http://doi.org/10.1016/j.virol.2006.03.052

32. Vallente R, Eichler E (2002) OPINION Segmental duplications and the evolution of the primate genome. Nat Rev Genet 3:65-72. https://doi.org/10.1038/nrg705

33. Peng T, Li Y (2009) Tandem exon duplication tends to propagate rather than to create de novo alternative splicing. Biochem Biophys Res Commun 383:163-166. https://doi.org/10.1016/j.bbrc.2009.03.162

34. Moore AD, Bjorklund AK, Ekman D, Bornberg-Bauer E, Elofsson A (2008) Arrangements in the modular evolution of proteins. Trends Biochem Sci 33:444-451. https://doi.org/10.1016/j.tibs.2008.05.008

35. Trump N, Cullup T, Verheij JB, Manzur A, Muntoni F, Abbs S, Jungbluth H (2012) X-linked myotubular myopathy due to a complex rearrangement involving a duplication of MTM1 exon 10. Neuromuscul 
Disord 22:384-388. https://doi.org/10.1016/j.nmd.2011.11.004

36. Rashid S, Breckle R, Hupe M, Geisler S, Doerwald N, Neesen J (2006) The murine Dnali1 gene encodes a flagellar protein that interacts with the cytoplasmic dynein heavy chain 1. Mol Reprod Dev 73:784-794. https://doi.org/10.1002/mrd.20475

37. Inaba A, Furuhata M, Morimoto K, Rahman M, Takahashi O, Hijikata M, Knowles MR, Keicho N (2019) Primary ciliary dyskinesia in Japan: systematic review and meta-analysis. BMC Pulm Med 19:135. http://doi.org/10.1186/s12890-019-0897-4

38. Kumar K, Taylor WE, Shen R, Stefan A, Matthew G, Fisher CE, Coucke PJ, Peter VH, Guy VC, Shalender B (1997) Complementary deoxyribonucleic acid cloning and characterization of a putative human axonemal dynein light chain gene 1. J Clin Endocr Metab 82:3047-3053.

http://doi.org/10.1210/jcem.82.9.4242

39. Liu ZB, Tu HQ, Kang YS, Xue YY, Ma DY, Zhao CT, Li HY, Wang L, Liu F (2019) Primary cilia regulate hematopoietic stem and progenitor cell specification through Notch signaling in zebrafish. Nat Commun 10:1839. https://doi.org/10.1038/s41467-019-09403-7

40. Kobayashi D, Asano-Hoshino A, Nakakura T, Nishimaki T, Ansai S, Kinoshita M, Ogawa M, Hagiwara $\mathrm{H}$, Yokoyama T (2017) Loss of zinc finger MYND-type containing 10 (zmynd10) affects cilia integrity and axonemal localization of dynein arms, resulting in ciliary dysmotility, polycystic kidney and scoliosis in medaka (Oryzias latipes). Dev Biol 430:69-79.

https://doi.org/10.1016/j.ydbio.2017.08.016

41. Kamiya R, Kurimoto E, Muto E (1991) Two types of Chlamydomonas flagellar mutants missing different components of inner-arm dynein. J Cell Biol 112, 441-447. http://doi.org/ 10.1083/jcb.112.3.441

42. Sun JJ, Wu ZH, Zhong T (2016) Cilia function in cell signaling and organ development. Sci Sin Vitae 46:354-362. https://doi.org/10.1360/N052016-00134

43. Samsa LA, Givens C, Tzima E, Stainier DY, Qian L, Liu J (2015) Cardiac contraction activates endocardial Notch signaling to modulate chamber maturation in zebrafish. Development 142:40804091. https://doi.org/10.1242/dev.125724

44. Mayr C (2019) What are 3' UTRs doing? Cold spring harb perspect Biol 11. https://doi.org/10.1101/cshperspect.a034728

45. Yazawa R, Hirono I, Aoki T (2005) Characterization of promoter activities of four different Japanese flounder promoters in transgenic zebrafish. Mar Biotechnol (NY) 7:625-633. https://doi.org/10.1007/s10126-005-0011-1

46. Yazawa R, Hirono I, Aoki T (2006) Transgenic zebrafish expressing chicken lysozyme show resistance against Bacterial Diseases. Transgenic Res 15:385-391. https://doi.org/10.1007/s11248006-0009-0

47. Kim B, Kim HM, Kang MK, Sohn DH, Han SJ (2020) 5'-UTR and ORF elements, as well as the 3'-UTR regulate the translation of Cyclin. Biochem Biophys Res Commun 527:968-973. https://doi.org/10.1016/j.bbrc.2020.04.151 
a

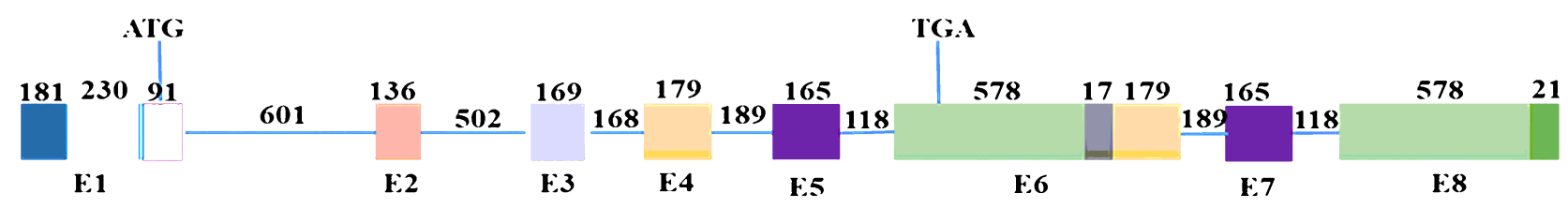

b
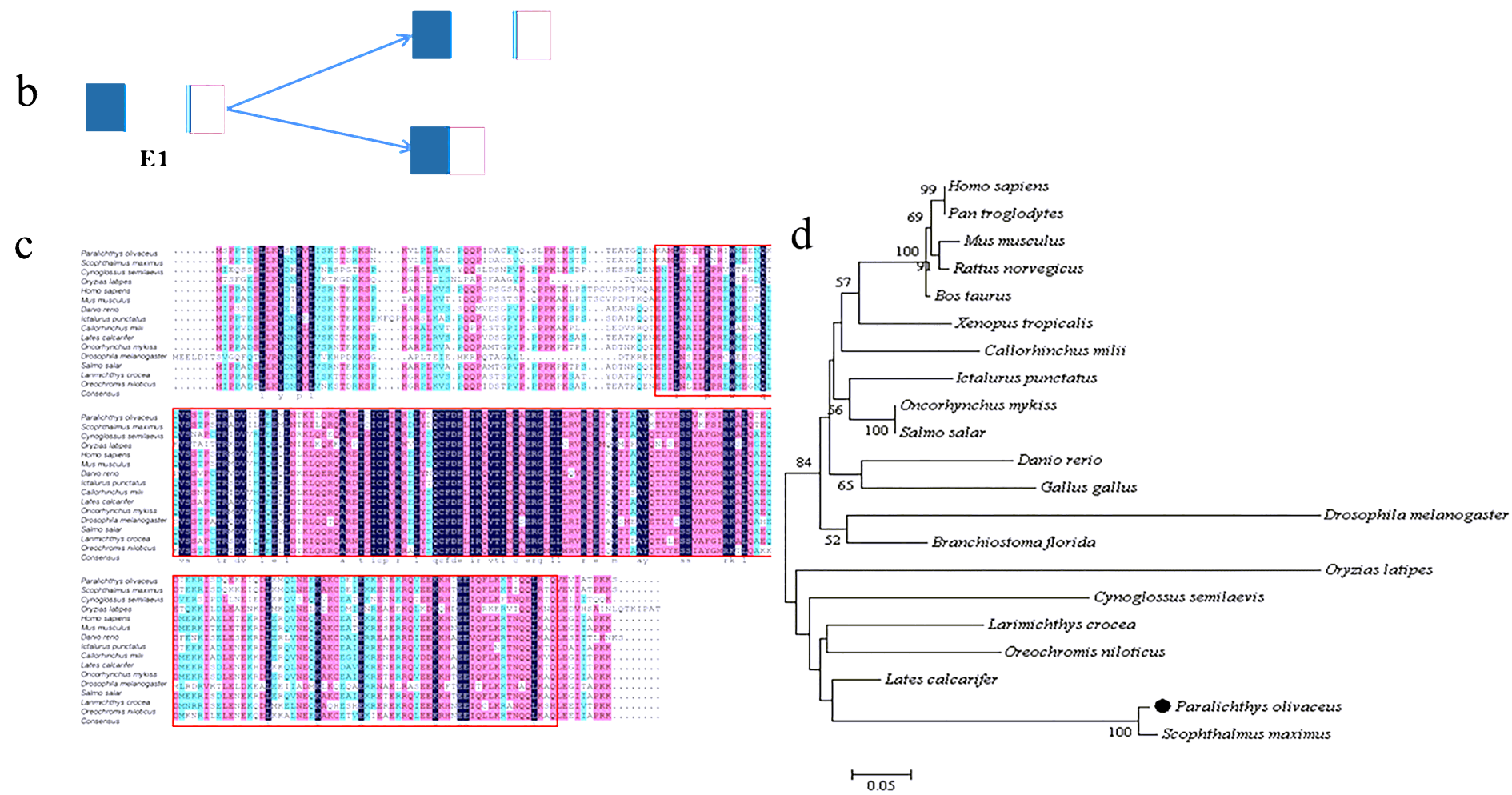

\section{Figure 1}

The analyses of gene structure and phylogenetic tree a, Genomic structure of the flounder dnali1. Colorful boxes indicate exons, and introns are between them. Modules of the same color in the last three exons mean the same sequence. $b$, Alternative splice of 5 ' UTR. There are two different alternatively splicing variants in 5'UTR. c, Multiple sequence alignment of the Dnali1. The identical and similar amino acids are highlighted in solid black and red, respectively. DNAMAN software was used to carry out the multiple sequence alignment. The red box denotes the conserved domain. $d$, Phylogenetic tree was built using the neighbor-joining method. Values at the branch points indicate the percentage of 1000 bootstrap replicates supporting the division. The flounder is marked. Animal scientific names and GenBank accession Nos. are listed in Table S1. 
${ }_{2 \text { 2kp }}$ 든

b ATGAGCCCACCAACAGATtCTCTCCTGAAATACAACAACCCGGTTTTGATCAGCAAGACCACAGACAGGAAATCAG ATAAGGT...........GCTGCCTTTAAGAGCATGCCCTCAGCAGCCCATTGACGCCTGCCCTGTTCAATCACTTC CCAAACTTAAATCCACCTCCACTGAGGCCACCGGGCAGGAAAATAAGGCCATGCTGGAAAACATCTTTCCAAACAG G......... ATTTGGATGGAAGAGAACCAAAAATGGCTGCAGCAAGTGTCCAGCACTCCTAGTACAAGAGCAGA TGTCATACTCCTCGAGGAAATGCTGAACACAAAGATTCTGCAGAGGCAGGCCAGAGAGACATGCATCTGTCCCATT CGCAGGGATCTCTACGACCAGTGCTTTG . . . . . A. ATGAGCTCATCCGACAGGTGACCATCAATTGTGCTGAG genomic part B-F

AGGGGTCTGCTGTTGTTGCGGGTTCGAGATGAGATTAAAATGACGATTGCTGCCTACAAGACACTGTATGAGAGCA GCGTTAAGTTCAGCATAAGGAAAGCACTGCAGACCGAGCAGAACAAAGCTGACACAGAGAAAAGGqtaagggctga gttaattagttatttatgaatgacccattcagaggaacaatgatacagttttctgtcaagcagaaagctccattt ctcttaatattaactaaaataatgatggtagcacattttactgaatagatcccgcttctgtaacctgctcatatga gtgtctcqttaatatatgtatctagATTTCGGATCAGAAGAAAGAGATACAAGACCTGAAGATGCAACTGAATGA AGAGAAAGCCAAATGTGATGAGATCGAAAAGAAAGAAAATGAAAAGCGACAGGTGGAGGAAAAGAAGCACACAGAA GAGATTCAGTTCCTTAAGAAAACCATTCAGCAACTCAGGgtgaggtttaatcatcacatataaaagaaacatgtt ttccaagcatcagtagtagccgtattgaagattggttgcccgtcttaagatttatatcaacctatttctatttctt ttcagACCCAGGTCGAAGTGATCGCCACACCGAAGAAAAGCTGAAAACTTAAAAACCCTGAGTTACCATTTCACCA TTTGTCTTGTGAAAACTTAACTGAGTGTAATTAGCAAATATTAATTTTTCTTCATGGCAAGTACAGTTCAAACTAT TACTGTACATTTCTGAACGCCAAGAGAAAGGAAGAAACAAGAAAAAGGATCAGAAGAAGAAGAGTGGGAACATGAC AAAGCTAATGGGCTCGGGAGAAGCTAAAGCTAGGCATGCTGGCACTTTGGTAGCACTAGCCACAAACAAAGATGTC CTGGCAGCAATAAATTAGCTTAACAGAATGGTCAACATGAGGTTCACCAAGCTTAGTGGGTCCCTCTTGAGCCTTA AATCTTCTTTATCAGATGTCTGCAAGCGGGTGTCTTCAAAAAAAGGCTGCCACTGAATCGCATGAGAGGCGATTCG AGGAGCGGGAGAGGAGGCATGATAGCCTAGCATCTCAGTACATACAACAACAAGCCAAACTCGATGACTTGGAGGC TCACTCAAAACATTTGCATAATTGGGATCAAGGAGATGCTGATGCTGGTACGTCTTACTTTTTCCCAGATGAGCTC ATCCGACAGGTGACCATCAATTGTGCTGAGAGGGGTCTGCTGTTGTTGCGGGTTCGAGATGAGATTAAAATGACGA TTGCTGCCTACCAGACACTGTATGAGAGCAGCGTTAAGTTCAGCATAAGGAAAGCACTGCAGACCGAGCAGAACAA AGCTGACACAGAGAAAAGGgtaagggctgagttaattagttattttatgaatgacccattcagaggaacaatgata cagttttctgtcaagcagaaagctccatttctcttaatattaactaaaataatgatgrtagcacattttactgaat agatcccqcttctgtaacctgctcatatgagtgtctcqtttaatatatgtatctagATTTCCAATCAGGAGAAAGA GATACAAGACCTGAAGATGCAACTGAATGAAGAGAAAGCCAAATGTGATGAGATCGAAAAGAAAGAAAATGAAAAG CGACAGGTGGAGGAAAAAAAGCACACAGAAGAGATTCAGTTCCTAAAGAAAACCATTCAGCAACTCAGGgtgagqt ttaatcatcacatataaaagaaaacatgttttccaagcatcagtagtagccgtattgaagattggttgcccgtctt aagatttatatcaacctattctattctttcagACCCAGGTCGAAGTGATCGCCACACCGAAGAAAAGCTGAAA ACTTAAAAACCCTCAGTTACCATTTCACCATTTGTCTTGTGAAAACTTAACTGAGTGTAATTAGCAAATATTAAAT TTTCTTCATGGCAAGTACAGTTCAAACTTTTACTGAACATTTCTGAACGCCAAGAGAAAGGAAGAAACAAGAAAAA GGATCAGAAGAAGAAGAGTGGGAACATGACAAAGCTAATGGGCTCGGGAGAAGCTAAAGCTAGGCATGCTGGCACT TTGGTAGCACTAGCCACAAACAAAGATGTCCTGGCAGCAATAAATTAGCTTAACAGAATGGTCAACATGAGGTTCA CCAAGCTTAGTGGGTCCCTCTTGAGCCTTAAATCTTCTTTATCAGATGTCTGCAAGCAGGTGTCTTCAAAAAAAGG CTGCCACTGAATCGCATGAGAGGCGATTCGAGGAGCTAGAGAGGAGGCATGATAGCCTAGCATCTCAGTACATAAA ACAACAAGCCAAACTCGATGAGTTGGAGGCTCACTCAAAACATTTGTATAATTGGGATCAAGGAGATGCTGATGCT GGTACCTCATTAAAATATCCAATTGG

genomic part B-R

\section{Figure 2}

Genomic sequences of dnali1 The red letters indicate the start codon and stop codon. The underlined nucleotides are the repeated sequences. Lowercase letters and “..." mean introns. The letters with box are specific primers for the genomic sequence confirmation. 

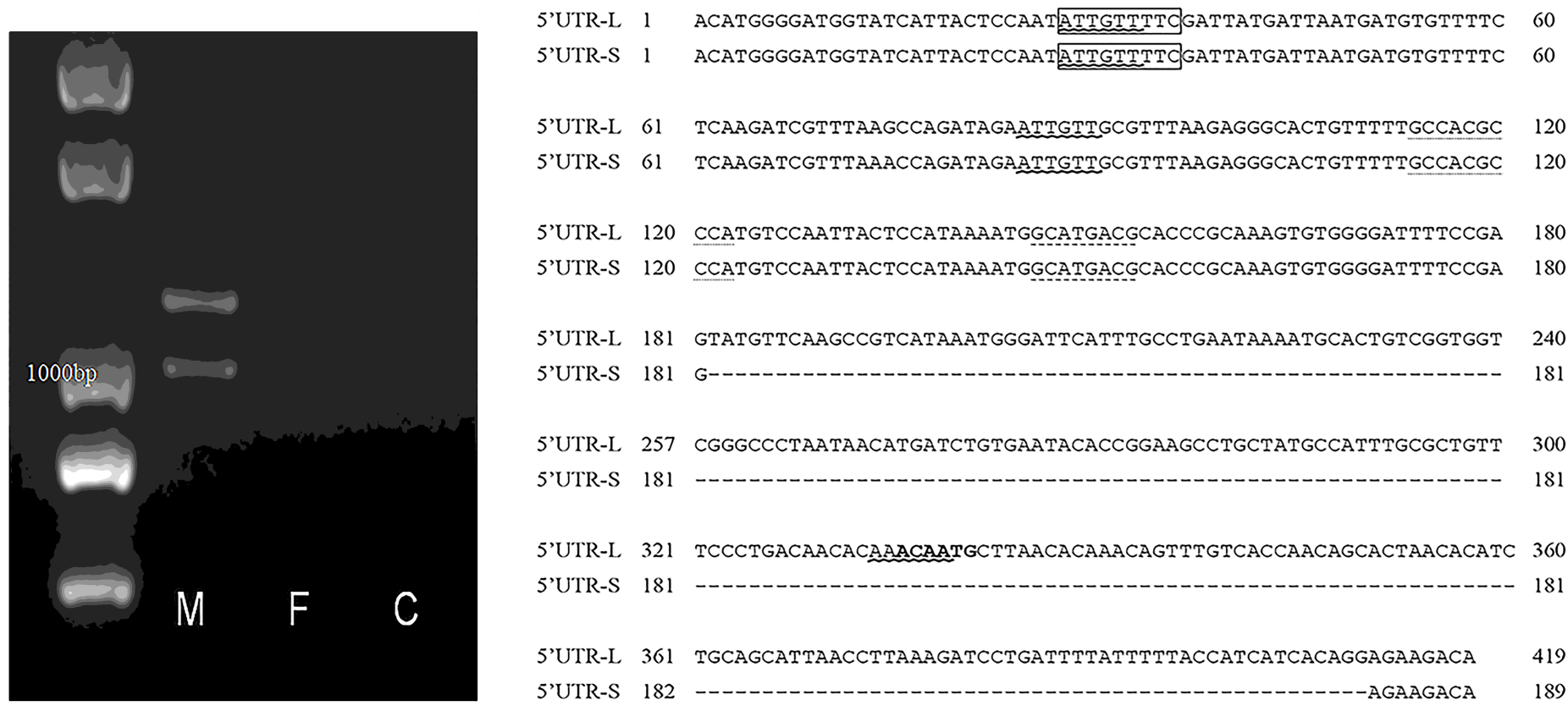

Figure 3

See the manuscript file for the complete figure caption.

a

$\begin{array}{llllllllllllll}\text { M } & \text { B } & \text { St } & \text { H } & \text { E } & \text { Mu } & \text { Sp } & \text { HK } & \text { I } & \text { K } & \text { G } & \text { Gi } & \text { L } & \text { NC }\end{array}$
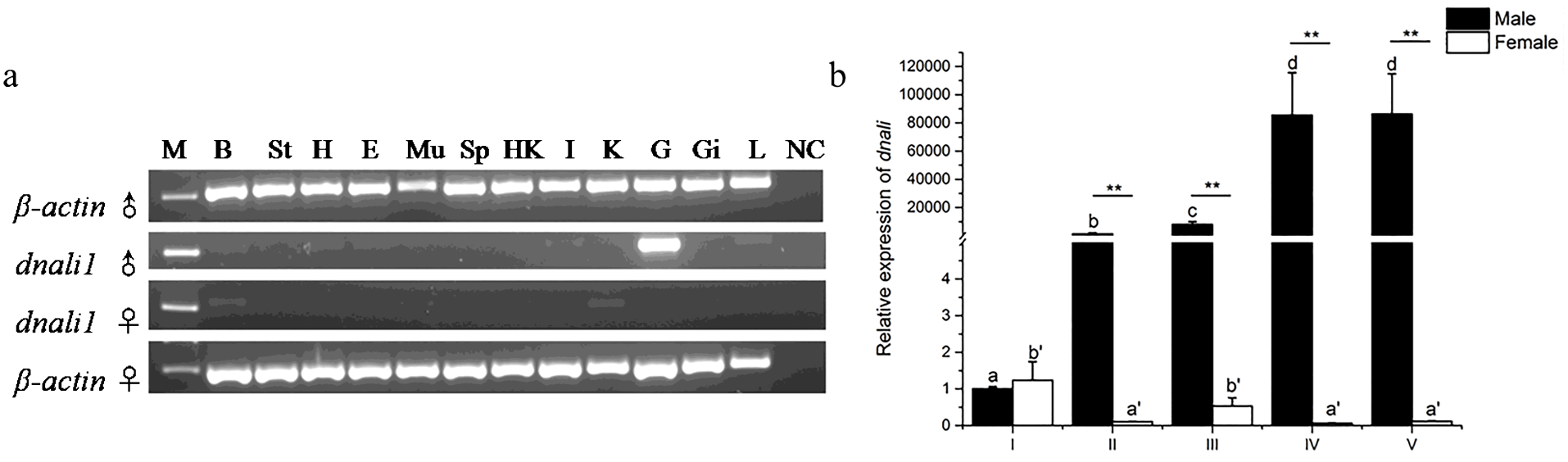

Gonadal development stage

\section{Figure 4}

Expression of the flounder dnali1 in different tissue and gonads a, Tissue distribution. $\mathrm{M}$, marker; $\mathrm{B}$, brain; St, stomach; H, heart; E, eye; Mu, muscle; Sp, spleen; HK, head kidney; I, intestine; K; kidney; G, testis/ovary; $\mathrm{Gi}$, gill; L, liver. NC, negative control. b, Expression in the gonads. Different letters indicate significant differences at gonadal development stages of the testis and ovary $(P<0.05)$. * represents significant difference between the testis and ovary at same gonadal development stage $(P<0.05)$; ** represents extremely significant difference $(P<0.01)$. 

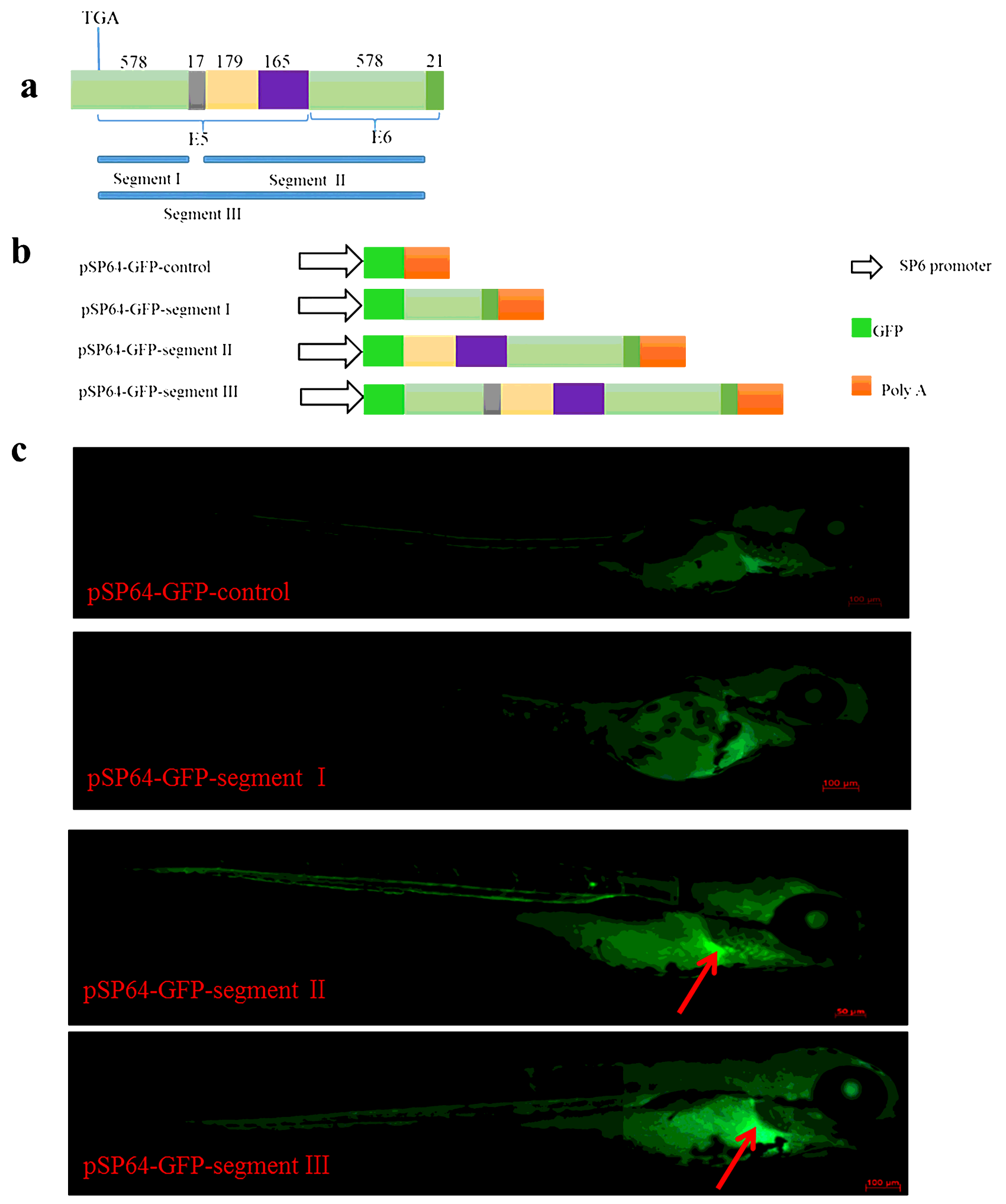

\section{Figure 5}

3'UTR structure, schematic illustration of constructed plasmids, and GFP expression regulated by the flounder dnali1 3'UTR in zebrafish a, 3'UTR structure. 3'UTR sequences could be divided into two parts: fragments $\nabla$ and $\otimes$. Fragment $\nabla$ represents the rest of the first repeat segment behind termination codon, fragment \contains the whole second 922 bp repeat segment, and fragment $₫$ represents whole 3'UTR $^{\prime}$ (fragments $\otimes$ and $\nabla$ ). b, Schematic illustration of the constructed plasmids. Different color boxes indicate 
elements in the plasmids. c, GFP expression in the control, fragment $\varangle$, fragment $\varangle$, and fragment $\otimes$ groups. The red arrow indicates positive signal.

\section{Supplementary Files}

This is a list of supplementary files associated with this preprint. Click to download.

- Fig.S1.tif

- SupplementaryTable.docx 